\section{Formação de avaliadores na modalidade educação a distância: necessidade transformada em realidade}

\section{The training of evaluators in the Distance- Learning Module: a need becomes a reality}

\author{
Luciana Santos Dubeux 1 \\ Gisele Cazarin2 \\ Ana Claudia Figueiró 3 \\ Luciana Caroline Albuquerque Bezerra4 \\ Marcos Barros 5 \\ Ângela Salvi6 \\ Dulcineide Oliveira7 \\ Gustavo Sampaio8
} 1-8 Grupo de Estudos de Gestão e Avaliação em Saúde (GEAS).
Diretoria de Pesquisa. Instituto Materno Infantil Prof. Fernando
Figueira-IMIP. Rua dos Coelhos, 300. Recife, PE, Brasil. CEP:
50.070-550. E-mail: lucianadubeux@imip.org.br.

\begin{abstract}
This article presents the proposal for the Basic Course in Health Evaluation of the Instituto Materno Infantil Prof. Fernando Figueira - IMIP - which aims to teach the main concepts and approaches adopted in this field of work, the use of distance learning principles and technology. The course forms an integral part of the National Policy for Institutionalization of Health Evaluation, with a view to training professionals occupying strategic positions in the three management levels of the Brazilian National Health Service, in various parts of the country. Given the expected profile of the health evaluator, the necessary qualifications for this professional were established and a teaching proposal centered on critical, reflective, autonomous learning and based on a dialogue between theory and the practical work of the student. To design the course, a multi-professional team was set up (including evaluators, teaching consultants, computer technicians and designers). The work was divided into the following stages: design and production of teaching materials, development of the website, training of tutors, and selection of students.
\end{abstract} Key words Distance learning, Health evaluation, Single Health System

\section{Resumo}

Será apresentada neste artigo a proposta do Curso Básico de Avaliação em Saúde do Instituto Materno Infantil Prof. Fernando Figueira - IMIP - voltada ao ensino dos principais conceitos e abordagens nesse campo de atuação, com o uso de fundamentos e da tecnologia de Educação à Distância. Este curso é parte integrante da Política Nacional de Institucionalização da Avaliação em Saúde, com vistas à formação de profissionais situados em posições estratégicas nas três instâncias gestoras do Sistema Único de Saúde, nas diversas regiões brasileiras. Considerando o perfil esperado para o avaliador em saúde foram estabelecidas as competências para este profissional e uma proposta pedagógica centrada no aprendizado crítico, reflexivo, autônomo e com base no dialogo entre a teoria e a vivência prática do aluno. Para a elaboração deste curso foi conformada uma equipe multiprofissional (avaliadores, consultores pedagógicos, técnicos de informática e diagramação), sendo realizadas as seguintes etapas: elaboração do material didático, desenvolvimento da página na rede, capacitação de tutores e seleção dos alunos.

Palavras-chave Educação a distância, Avaliação em saúde, Sistema Único de Saúde 


\section{Introdução}

As iniciativas recentes no setor saúde visando à qualificação dos profissionais e das práticas avaliativas não são movimentos isolados do país, mas podem ser verificadas também nos países mais desenvolvidos. E tanto lá quanto aqui, fatores como a modernização da administração pública, a adoção de gestão por resultados, a introdução de mecanismos regulatórios e o requerimento da realização de avaliação de programas por organismos financiadores têm sido apontados como propulsores desse interesse crescente. $\mathrm{Na}$ experiência desses países aparece como denominador comum o investimento na construção de competência em avaliação, e como objetivo o seu uso na tomada de decisão. ${ }^{1,2}$

No Brasil, após a consolidação das conquistas legais do Sistema Único de Saúde (SUS) - entre as quais podemos citar: novos procedimentos administrativos na gestão do sistema; descentralização das responsabilidades, ações e recursos; rápida incorporação de tecnologia; exigência cada vez maior do controle de gastos em saúde; e, com mais freqüência e contundência, o acompanhamento dos investimentos pelos organismos financiadores externos tem havido um interesse crescente em avaliação de políticas, programas e serviços de saúde. Mais recentemente, a oferta de uma atenção à saúde com qualidade e resolubilidade tem-se constituído em uma das principais preocupações dos gestores do SUS, e a avaliação de programas tem sido frequentemente referida como uma importante ferramenta para qualificação das ações e práticas de saúde. Porém, para que sejam capazes de cumprir sua função, os interessados no campo da avaliação devem encarar o desafio de melhorar a imagem e a credibilidade da função avaliativa e, de fato, garantir o maior e melhor uso das avaliações pelos tomadores de decisão. ${ }^{3}$ Como estratégia para construção de capacidade em avaliação, cuja formação profissional de avaliadores é um dos pilares, o Ministério da Saúde, no âmbito da Política de Institucionalização da Avaliação em Saúde, implementou, entre outras iniciativas, o Programa Interinstitucional para Formação de Avaliadores em Atenção Básica de Saúde, com vistas à formação de profissionais situados em posições estratégicas nas três instâncias gestoras do Sistema de Saúde, nas diversas regiões brasileiras. Tendo sido desenvolvido em colaboração com instituições parceiras - Instituto Materno Infantil Prof. Fernando Figueira (IMIP), Instituto de Saúde Coletiva da Universidade Federal da Bahia (ISC/UFBA), Escola Nacional de Saúde Pública Sérgio Arouca da Fundação Oswaldo Cruz
(ENSP/FIOCRUZ) e Universidade de Montreal foram definidas conjuntamente as diretrizes $\mathrm{e}$ competências necessárias para essa formação, bem como se estabeleceu a oferta de cursos de avaliação em saúde nos níveis básico, intermediário e avançado. Optou-se pela proposta de formação utilizando processo de educação a distância e coube ao IMIP a elaboração e oferta do curso básico, cuja proposta em desenvolvimento será apresentada neste artigo.

\section{Educação a Distância como uma estratégia e não apenas uma alternativa}

A realização de cursos de formação profissional (técnico, graduação e pós-graduação) na modalidade de educação a distância vem se consolidando no Brasil como estratégia eficaz para atender a necessidade social da universalização do acesso ao ensino de qualidade. Para atender a essa demanda, o Ministério da Educação constituiu a Secretaria de Educação a Distância (SEED), voltada para a ampliação e interiorização da oferta de ensino superior gratuito e de qualidade no país, representando a clara intenção de investir na educação a distância e nas novas tecnologias como uma das alternativas para democratizar e elevar o padrão da educação brasileira. $^{4}$

Segundo Moran, ${ }^{5}$ essa modalidade de educação refere-se a um processo de ensino-aprendizagem mediado por tecnologias, onde estudantes e tutores estão separados física, espacial e/ou temporalmente, porém, poderão estar conectados mediante tecnologias, sobretudo as telemáticas, a exemplo da Internet, ou podendo utilizar outros meios, tais como: correio, rádio, televisão, vídeo, CD-ROM, telefone, fax entre outras tecnologias semelhantes.

O principal objetivo nas propostas de educação a distância é o de promover uma aprendizagem autônoma. A linguagem utilizada é diferenciada, pois procura dar ênfase a processos pedagógicos autônomos e interativos. Nesse sentido, o papel primordial dos tutores é de manter a motivação, para que os alunos sejam instigados e desafiados a enveredar pela aventura da construção da autonomia do próprio conhecimento. ${ }^{5,6}$ Dessa forma, preconizase que o tutor desenvolva estratégias de ensino com foco no aluno, o qual deve se identificar como principal responsável pelo aprendizado. Seguindo esse raciocínio, o ambiente educacional virtual deve, também, promover a criação de situações que estimulem os alunos ao desenvolvimento de atividades que valorizem, além da autonomia, a criatividade e o 
pensamento reflexivo, como fundamentos básicos para o processo de ensino-aprendizagem.

\section{O Curso Básico de Avaliação em Saúde}

\section{Perfil do avaliador em saúde}

Para a formação de avaliadores, tomou-se como ponto de partida as proposições sobre as competências esperadas para os profissionais dedicados à avaliação de programas sociais (incluindo nessa denominação políticas, programas, projetos, serviços ou práticas) de países que vêm se dedicando há mais tempo sobre o tema. ${ }^{3,7,8}$ A partir dessas reflexões, buscou-se identificar o perfil esperado desse profissional, considerando a realidade e necessidade do campo da saúde no Brasil, e as seguintes competências foram relacionadas como requisitos que os avaliadores em saúde devem desenvolver na sua formação: atitude ética; respeito aos valores e crenças dos diversos atores sociais; competência teórica e metodológica sobre o tema; atitude investigativa a partir da reflexão dos modelos e matrizes confrontados com a realidade; visão interdisciplinar e atuação de forma cooperativa e sinérgica; comunicação interativa e habilidade de negociação, entre outros.

\section{Objetivos}

O objetivo principal é a capacitação de profissionais de saúde que atuam em organizações públicas de todo Brasil, acerca dos principais conceitos e abordagens metodológicas em avaliação de programas de saúde. Como objetivos específicos espera-se que o aluno seja capaz de: selecionar e descrever os problemas de saúde prioritários e as intervenções/programas existentes para atuar sobre os mesmos; conhecer os objetivos, tipos e conceitos básicos em avaliação; identificar os principais desenhos de estudos em avaliação e avaliar a qualidade deles; reconhecer os passos fundamentais para o desenvolvimento de avaliação de intervenções/programas em saúde; refletir sobre a prática profissional e a cultura da avaliação nas instituições de saúde; identificar os aspectos éticos envolvidos na prática avaliativa; e, por fim, realizar um estudo de avaliabilidade de um programa de saúde.

\section{Proposta pedagógica}

A estratégia pedagógica utilizada no curso é dire- cionada a estimular o estudo autônomo, bem como o aprendizado e a reflexão baseada no diálogo entre teoria e prática, trazendo a realidade do local de trabalho do estudante como parte integrante do processo ensino/aprendizagem. Corroborando esses princípios, Rocha et al. ${ }^{9}$ afirmam que o ensino com foco na rotina do sistema de saúde, na qual o estudante está inserido, o auxilia a trazer sua realidade para o contexto das atividades curriculares, permitindo exercitar a elaboração de informações para compreensão do sistema envolvido.

O curso foi desenvolvido seguindo as competências profissionais mencionadas anteriormente. Considerou-se que essas são parte integrante de um sistema de ação complexo que envolve conhecimentos, habilidades, emoções e atitudes, bem como estratégias e rotinas para aplicação apropriada desses. $^{8}$

O desenvolvimento das competências será estimulado durante os Módulos de Aprendizagem, definidos como uma rede de conhecimentos teóricopráticos organizados em uma estrutura pedagógica orientada por objetivos de aprendizagem em função de um conjunto articulado de conteúdos. Os Módulos contemplarão Unidades Pedagógicas e Seqüência de Atividades, organizadas de maneira lógica, com o objetivo de abordar um ou mais conteúdos, constituindo um ciclo de: prática que corresponde às competências ou atribuiçõos; discussão, sistematização e teorização, possíveis mediante o desenvolvimento dos objetivos propostos nos módulos, unidades pedagógicas e seqüência de atividades que subsidiarão a volta à prática com a formação profissional baseada nas competências esperadas (Figura 1).

Nesse curso foram previstos sete Módulos de Aprendizagem, sendo o módulo de integração de caráter instrutivo e de aproximação entre os estudantes e tutores, os módulos de 1 a 5 abrangendo o conteúdo programático do curso, e o módulo final para a consolidação das atividades desenvolvidas e apresentação do produto final do curso. O curso disponibiliza, ainda, um glossário específico com termos da área de avaliação.

A seguir serão apresentados o detalhamento dos conteúdos dos Módulos de aprendizagem, as respectivas Unidades Pedagógicas e carga horária prevista (Tabela 1).

Esses conteúdos serão abordados em 85 horas, distribuídos em 17 semanas, sendo sugerido que o estudante dedique ao menos cinco horas semanais para realização das atividades. Cada Módulo apresenta um tempo previsto para sua realização que serve de orientação ao aluno. 

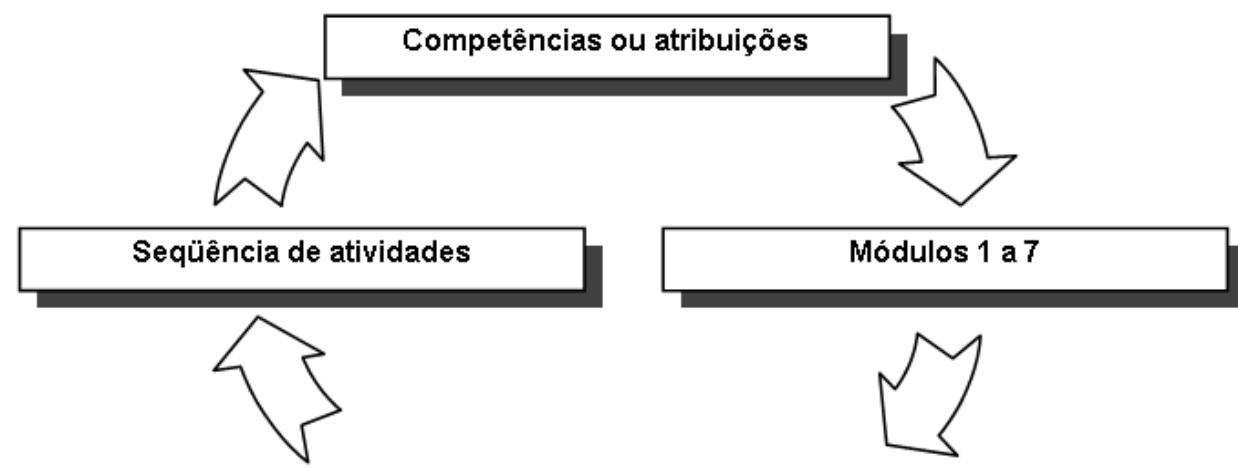

Unidades pedagógicas

Tabela 1

Distribuição dos Módulos de Aprendizagem e Unidades Pedagógicas do Curso Básico de Avaliação em Saúde.

\section{Módulos (M)}

Módulo Integração: conhecendo o ambiente virtual e os participantes

M1: Descrevendo problemas e intervenções de saúde

M2: Conceitos e métodos de avaliação

M3: Etapas da avaliação I

M4: Etapas da avaliação II

M5: Prática profissional em avaliação

Módulo Final: Desenvolvendo o estudo de avaliabilidade

\section{Unidades Pedagógicas (UP)}

$---$

\section{Revisando o conceito e as práticas da atenção básica} Identificação e descrição de problemas de saúde Identificação e descrição de intervenções de saúde

Conceitos e tipos de avaliação

Desenhos de estudos de avaliação

Monitoramento e Avaliação

Meta-avaliação

Identificação dos interessados na avaliação

Modelo Lógico do programa (ML)

Foco do projeto da avaliação

Desenho da avaliação

Justificativa das conclusões

Uso e compartilhamento das lições aprendidas

Cultura de avaliação

Reconhecer-se como avaliador

1 Etapas de um estudo de avaliabilidade 
O conjunto dos temas/conteúdos será apresentado através de recursos didáticos específicos: textos, estudos de casos, situações-problema e exercícios. Esses recursos serão vivenciados pelos estudantes através de ferramentas tecnológicas disponíveis no ambiente virtual de aprendizagem: fórum, bate-papo ou chat, diário e tarefa com envio de arquivo. O fórum representa uma discussão textual on-line em modalidade assíncrona, onde a comunicação não-simultânea favorece reflexões e debates por período maior de tempo. De forma oposta, o bate-papo e a realização de um diálogo textual on-line em modalidade síncrona, oferecem subsídios para uma comunicação rápida e instantânea entre tutores e estudantes, sendo ainda estratégicos para promover a integração dos estudantes. O diário é o espaço de reflexão individual sobre o processo de aprendizagem no curso por parte do estudante, que no caso desse curso permitirá a elaboração do estudo de avaliabilidade a ser apresentado como produto final. Por sua vez, a tarefa com envio de arquivo permite que cada estudante envie um único arquivo solicitado pela atividade.

\section{Proposta de avaliação}

O ambiente virtual de aprendizagem a ser utilizado permite a geração de relatórios de acompanhamento do aluno durante o curso. Essas informações servirão de base para que o tutor realize uma avaliação processual do desempenho do estudante. Será considerada a atuação do aluno nos fóruns de discussão, diário, bate-papo e tarefa com envio de arquivo, mediante a observação dos seguintes critérios de avaliação: cumprimento dos prazos, participação ativa, capacidade de articulação entre teoria e prática, capacidade de análise crítica e interpretação dos conteúdos; exposição de idéias e informações de forma sintética, clara e organizada, interação com colegas e tutores; flexibilidade para receber críticas. Ao final do curso, o conceito individual do aluno será calculado através da média entre a avaliação dos módulos e a avaliação do produto final do curso.

\section{Fases de desenvolvimento do curso}

A conformação de uma equipe multiprofissional, com a participação de especialistas no campo da avaliação em saúde, consultores pedagógicos, técnicos de informática e diagramação, foi primordial para a elaboração do curso, cuja construção ocorreu a partir da realização das seguintes etapas: elaboração do material didático, desenvolvimento da página na rede, capacitação de tutores e seleção dos alunos.

Coube aos especialistas em avaliação em saúde, sob orientação de consultores pedagógicos, desenvolverem o material didático do curso, baseando-se na proposta pedagógica anteriormente descrita. No intuito de viabilizar o ensino a distância, selecionouse o Moodle ${ }^{\circledR}$ (Modular Object Oriented Distance Learning), como ambiente virtual de aprendizagem. O Moodle ${ }^{\circledR}$ é um software livre que atua como ferramenta de processo dinâmico de aprendizagem por meio de trocas, orientado por uma filosofia de "pedagogia social construcionista", ou seja,

[...] um grupo de pessoas construindo coisas umas para as outras, criando de maneira colaborativa uma pequena cultura de coisas compartilhadas com significados compartilhados". (Pulino Filho). ${ }^{10}$

Os tutores foram capacitados para o desenvolvimento das dimensões pedagógica e tecnológica do curso, uma vez que a dimensão teórica (competência no campo da avaliação em saúde) foi um dos requisitos para a tutoria. Neste sentido, a formação teve como foco a prática docente a distância e aspectos do manuseio do ambiente virtual utilizado (Moodle $^{\circledR}$ ).

Os alunos selecionados são oriundos de todas as regiões do país, contemplando gestores e coordenadores de Secretarias estaduais e municipais de Saúde, instituições de ensino e pesquisa, Ministério da Saúde. Essa seleção buscou atender aos propósitos de construção de capacidade avaliativa nas organizações de saúde do SUS no país, de acordo com a Política Nacional.

\section{Considerações finais}

A formação de avaliadores utilizando a abordagem de Educação a Distancia, iniciada com a experiência do Curso Básico de Avaliação em Saúde oferecido pelo IMIP, se soma às iniciativas para a institucionalização da avaliação em saúde em andamento no país. A relevância deste projeto se destaca pela possibilidade de abrangência regional e nacional na qualificação dos profissionais interessados em desenvolver a função avaliativa, oportunizando o acesso ao ensino sem prejuízo da qualidade da formação. Internamente tem-se constituído numa oportunidade para consolidação dessa modalidade de ensino-aprendizagem com vistas à elaboração e oferta de cursos dessa natureza em outras áreas temáticas, com a conformação de uma equipe multiprofissional e a apropriação tecnológica necessária para viabilização deste processo. 


\section{Referências}

1. Sanders JR. Presencial address: on mainstreaming evaluation. Am J Eval. 2002; 23: 253-9.

2. Stevenson JF, Florin P, Mills DS, Andrade M. Building evaluation capacity in human service organizations: a case study. Eval Prog Plan. 2002; 25: 233-43.

3. Centre of Excellence in Evaluation. Treasury Board of Canada Secretariat. Improving the professionalism of evaluation. Available from: URL: http://www.tbs-sct.gc.ca/ eval/dev/Professionalism/profession_e.asp. [2006 Jan 21].

4. Brasil. Ministério da Educação. Curso piloto. Disponível em URL: http://www.uab.mec.gov.br/cursopiloto.php [2005 out 21].

5. Moran JM. O que é educação a distância. Disponível em URL: http://umbu.ied.dcc.ufmg.br/moodle/file.php/117/ Nivel_0/Conteudo/O_que_educao_a_distancia.pdf. [2005 out 21].
6. Pallof RM, Pratt K. O aluno virtual: um guia para trabalhar on-line. Porto Alegre: Artmed; 2004.

7. English B. Competencies for evaluation practitioners: where to from here? Eval J Australasia. 2002; 2: 13-5.

8. Stevahn L, King JA, Ghere G, Minnema J. Establishing essential competencies for program evaluators. Am J Eval. 2005; 26: 43-59.

9. Rocha JSY, Caccia-Bava MCG, Rezende CEM. Pesquisaaprendizagem no ensino da política e gestão de saúde: relato de uma experiência com e-Learning. Rev Bras Educ Med. 2006; 30: 73-8.

10. Pulino Filho AR. Ambiente de aprendizagem Moodle UnB: manual do professor. Disponível em URL: http://aprender.unb.br/mod/resource/view.php?inpopup=tru e\&id=1822 [2006 out 10]. 